\title{
Soluble urokinase plasminogen activator receptor levels are associated with severity of fibrosis in nonalcoholic fatty liver disease
}

\author{
Christopher Sjöwall, Klara Martinsson, Kristina Cardell, Mattias Ekstedt and Stergios \\ Kechagias
}

\section{Linköping University Post Print}

\section{Tweet}

N.B.: When citing this work, cite the original article.

Original Publication:

Christopher Sjöwall, Klara Martinsson, Kristina Cardell, Mattias Ekstedt and Stergios Kechagias, Soluble urokinase plasminogen activator receptor levels are associated with severity of fibrosis in nonalcoholic fatty liver disease, 2015, Translational research : the journal of laboratory and clinical medicine, (165), 6, 658-666.

http://dx.doi.org/10.1016/j.trs1.2014.09.007

Copyright: Elsevier

http://www.elsevier.com/

Postprint available at: Linköping University Electronic Press

http://urn.kb.se/resolve?urn=urn:nbn:se:liu:diva-112698 


\section{Full-length article}

Title: Soluble urokinase plasminogen activator receptor levels are associated with severity of fibrosis in non-alcoholic fatty liver disease

Authors: Christopher Sjöwall a, ${ }^{*}$ Klara Martinsson a, Kristina Cardell b, Mattias Ekstedt c, Stergios Kechagias $d$

${ }^{a}$ Rheumatology/AIR, Department of Clinical and Experimental Medicine,

b Infectious Diseases, Department of Clinical and Experimental Medicine,

${ }^{c}$ Gastroenterology and Hepatology, Department of Clinical and Experimental

Medicine,

d Gastroenterology and Hepatology, Department of Medical and Health Sciences, Linköping University, Sweden.

\section{* Corresponding author at:}

Rheumatology Unit, University Hospital, SE-581 85 Linköping, Sweden.

E-mail address: christopher.sjowall@liu.se

Telephone +46101032416

Fax: +46101031844

Running head: suPAR associates with fibrosis in NAFLD

Key words: biomarker, fibrosis, inflammation, cirrhosis, liver disease, NAFLD, hepatitis C 
Conflicts of interest: All authors have read the journal's policy on conflicts of interest and have none to declare.

Word count: 2939

\section{Abbreviations}

APRI = AST-to-platelet ratio index; ALT = alanine aminotransferase; AST $=$ aspartate aminotransferase; AUC = area under the curve; $\mathrm{CRP}=\mathrm{C}$-reactive protein; $\mathrm{D}_{\mathrm{I}}=$ domain $\mathrm{I}$; $\mathrm{D}_{\mathrm{II}}=$ domain II; DIII = domain III; ELISA = enzyme-linked immunosorbent assay; GUCI = Göteborg University Cirrhosis Index; HCV = hepatitis C virus infection; HC = healthy controls; HSC = hepatic stellate cells; NAFLD = non-alcoholic fatty liver disease; NAS = NAFLD activity score; SLE = systemic lupus erythematosus; suPAR = soluble urokinase plasminogen activator receptor; $\mathrm{uPAR}=$ urokinase plasminogen activator receptor 


\section{Abstract}

The identification of individuals with severe liver fibrosis among patients with chronic liver disease is of major importance when evaluating prognosis, potential risk for complications, and when deciding treatment strategies. Although percutaneous liver biopsy is still considered "gold standard" for staging of liver fibrosis, attempts to find reliable non-invasive markers of liver fibrosis are frequent. Inflammation is essential for the progression of fibrosis. The urokinase plasminogen activator (uPA) and its receptor (UPAR) have been associated with hepatic inflammation and fibrosis in mice. High serum concentrations of soluble uPAR (suPAR) are suggested to be involved in inflammation, tissue remodeling and cancer metastasis. Here, we evaluated serum suPAR as a non-invasive test to detect liver fibrosis in 82 well-characterized nonalcoholic fatty liver disease (NAFLD) patients, and in 38 untreated patients with chronic hepatitis C (HCV) infection at the time of their first liver biopsy. suPAR levels were increased in chronic liver disease compared to blood donors ( $p<0.001)$. HCV patients had higher suPAR concentrations than NAFLD patients $(p<0.002)$. suPAR levels were associated with the severity of fibrosis, particularly in NAFLD, but did not correlate with inflammation. Regarding the performance in predicting severity of fibrosis, suPAR was essentially as good as other commonly used non-invasive fibrosis scoring systems. The results in HCV confirm previous observations. However, this is the first study to investigate suPAR as a biomarker in NAFLD and the results indicate that suPAR may constitute a severity marker related to fibrosis and prognosis rather than reflecting inflammation. 


\section{Introduction}

Hepatic fibrogenesis is a maladaptive wound-healing process that occurs in response to persistent, injurious stimuli affecting the hepatocytes. This results in a stereotypical chronic inflammatory response leading to hepatic stellate cell (HSC) activation that produces an accumulation of extracellular matrix complexes. ${ }^{1}$ The crosslinking of collagen fibrils within the extracellular matrix leads to fibrous scar formation and eventual distortion of the hepatic architecture. ${ }^{1}$ The presence and stage of liver fibrosis is of paramount importance in patients with chronic liver disease when deciding treatment strategies, response to therapy, prognosis, and the potential risk for complications.

The "gold standard" for staging liver fibrosis is still percutaneous liver biopsy. ${ }^{2}$ However, important limitations to biopsy exist. Significant complications, requiring hospital admission or prolonged hospital stay, occur in $1-5 \%$ of patients, and mortality has been reported to range between $0.1-1$ in 1000 patients. ${ }^{3}$ In addition, liver biopsy has two major limitations: sampling error and inter-observer variability. A needle liver biopsy only represents 1:50 000 of the total organ, i.e. the potential for sampling error remains substantial. Autopsy and laparoscopy studies have demonstrated that cirrhosis is missed on a single blind liver biopsy in between $10-30 \%$ of cases. ${ }^{4,5}$ The intra- and inter-observer variability may range from $15 \%$ to as high as 33\% for determining fibrosis stage. ${ }^{5}$

The attempts to find reliable non-invasive markers of liver fibrosis have recently increased dramatically as a result of the evolving novel therapies for hepatitis $\mathrm{C}$ and B.6,7 By analogy, a considerable interest has arisen in extending this work into the field of 
non-alcoholic fatty liver disease (NAFLD) because of its increased prevalence. ${ }^{8,9}$ Moreover, recent long term studies suggest that development of fibrosis in NAFLD has an important prognostic significance. ${ }^{10}$

The soluble urokinase plasminogen activator receptor (suPAR) constitutes the circulating form of the glycosylphosphatidylinositol-linked membrane protein urokinase-type plasminogen activator receptor (UPAR; CD87), and is involved in inflammation, tissue remodeling and cancer metastasis. ${ }^{11,12}$ uPAR is expressed by a wide range of immune cells whereas suPAR can be detected in different body fluids, including serum and plasma. ${ }^{11-17}$ Cell-surface uPAR expression is up-regulated upon stimulation with growth factors and cytokines such as interleukin-1 $1 \beta$ and tumor necrosis factor. ${ }^{18,19}$ The full length suPAR shed from the cell surface contains three domains (DI-III), and suPAR can occur in different cleaved forms consisting of only $\mathrm{D}_{\mathrm{I}}$ or $\mathrm{D}_{\mathrm{II}-\mathrm{III}}$, with partly divergent biological functions. ${ }^{12,20}$ Increased levels of suPAR have been found to predict disease outcome in various forms of cancer and infectious diseases. ${ }^{21,22}$ Furthermore, suPAR blood levels correlate with markers of organ dysfunction such as creatinine, urea, cystatin C, bilirubin and albumin in the severely ill. ${ }^{21}$ In chronic liver disease with progressive liver fibrosis/cirrhosis, suPAR has also been shown to be elevated and correlate with poor prognosis. ${ }^{23-25}$ In patients with systemic lupus erythematosus (SLE), suPAR seems to reflect organ damage/disease severity rather than ongoing inflammation. ${ }^{26}$

The main aim of the present study was to evaluate serum suPAR as a non-invasive test to detect liver fibrosis in patients with NAFLD and chronic hepatitis C virus infection (HCV), respectively. The secondary aim was to compare the diagnostic performance of 
suPAR with a number of simple non-invasive tests previously reported to be of clinical value in identifying advanced liver fibrosis.

\section{Methods}

\section{Patients and controls}

The basis for this investigation is formed by patients referred for evaluation to the Department of Gastroenterology and Hepatology or to the Department of Infectious Diseases at Linköping University Hospital because of persistently ( $>6$ months) elevated serum aminotransferases and/or alkaline phosphatase. A diagnostic work-up was performed including physical examination, laboratory investigations and liver biopsy to identify toxic, metabolic, viral and autoimmune liver diseases. Relevant clinical and laboratory data were collected at the time of liver biopsy. 82 patients who reported average weekly alcohol consumption $<140 \mathrm{~g}$ were diagnosed with biopsy-proven hepatic steatosis without any other concomitant liver disease or medication associated with fatty infiltration of the liver. These subjects constitute the NAFLD study group. 38 untreated patients who underwent liver biopsy were diagnosed with HCV based on serologic and genotypic findings and were subsequently included in the HCV study group.

In addition, 100 blood donors ( 50 men, 50 women; mean age 45.8 years; range 22-70) without ongoing medication served as healthy controls (HC) for the suPAR analyses. Subjects with NAFLD and HCV had blood drawn immediately prior to liver biopsy. Sera were kept frozen at $-70^{\circ} \mathrm{C}$ until analysis. 


\section{Histopathology}

Liver biopsies of all subjects were performed percutaneously with ultrasound guidance and $1.6 \mathrm{~mm}$ Biopince needles on an outpatient basis. Global inflammation grade (0-4) and fibrosis stage (0-4) was assessed according to the standardized semi-quantitative histological scoring system developed by Batts and Ludwig. ${ }^{27}$ Portal inflammation was reported separately (0-4) as described by Knodell et al. ${ }^{28}$ Histopathological activity in NAFLD was assessed with the NAFLD activity score (NAS), which is the unweighted sum of steatosis (0-3), lobular inflammation (0-3), and hepatocellular ballooning (0-2), ${ }^{29}$ and steatosis was also assessed quantitatively as described by Franzén et al. ${ }^{30}$

\section{Scoring of fibrosis}

In Table II, the 4 non-invasive fibrosis scoring systems that were used in the study are presented; the NAFLD fibrosis score, the AST-to-platelet ratio index (APRI), the BARD score and the Göteborg University Cirrhosis Index (GUCI) (Table II). ${ }^{31-34}$

\section{Routine laboratory measures}

All biochemical assessments were performed in routine at the Department of Clinical Chemistry, Linköping University Hospital and included albumin, alkaline phosphatase (ALP), aspartate aminotransferase (AST), alanine aminotransferase (ALT), highsensitivity C-reactive protein (hsCRP), $\gamma$-glutamyl transferase (GGT), bilirubin, prothrombin complex international normalized ratio (PK-INR), and platelet count. Furthermore, in the NAFLD patients fasting plasma glucose, and fasting serum insulin was analyzed. Insulin resistance was assessed by the quantitative insulin sensitivity check index (QUICKI; [1/(log(fasting insulin $\mu \mathrm{U} / \mathrm{mL})+\log ($ fasting glucose $\mathrm{mg} / \mathrm{dL}))]) .{ }^{35}$ 
The presence of metabolic syndrome and body mass index (BMI) were recorded at blood sampling (body weight $\left.[\mathrm{kg}] / \mathrm{m}^{2}\right) \cdot{ }^{36}$

\section{Serotyping, RNA detection/quantification, and genotyping}

The anti-HCV Microparticle Enzyme ImmunoAssay (Abbott, Abbott Park, IL, USA) was used for HCV serology screening. Confirmation was performed with a recombinant immunoblot assay. RNA was extracted from plasma and initially analyzed with an inhouse nested polymerase chain reaction, followed by the Cobas Amplicor Hepatitis C virus test version 2.0 (Roche Diagnostics, Branchburg, NJ, USA). HCV-RNA was quantified by the branched DNA technique (Quantiplex HCV RNA version 2.0; Chiron Corp., Emeryville, CA, USA). HCV genotypes were determined after hybridization of biotin-labeled polymerase chain reaction products to oligonucleotide probes bound in strips on nitrocellulose membranes (Inno-Lipa, Innogenetics, Brussels, Belgium). All analyses were performed at Linköping University Hospital except for HCV genotyping, which was performed at the Department of Microbiology, Karolinska University Hospital, Stockholm, Sweden.

sUPAR assay

Sera were analyzed in duplicate for suPAR levels using suPARnostic ${ }^{\circledR}$ standard ELISA from Virogates (Birkeröd, Denmark). Briefly, serum samples, standard and curve control samples were mixed with peroxidase-conjugated detection antibody and transferred to pre-coated plates. Following $1 \mathrm{~h}$ incubation in room temperature (RT) the wells were washed and TMB substrate added. The plates were incubated for $20 \mathrm{~min}$ at RT, stop solution was added and the plates read at $450 \mathrm{~nm}$ (Sunrise ${ }^{\mathrm{TM}}$ microplate reader with Magellan $^{\mathrm{TM}}$ software, Tecan, Grödig, Austria). ${ }^{26}$ 


\section{Statistics}

Differences in suPAR levels between groups were analyzed using ANOVA followed by the post-hoc test Tukey. Differences between groups of diagnoses were analyzed with Ttest or Chi-2, were appropriate. Correlation analyses were performed using Pearson's correlation test. Receiver-operating characteristics (ROC) curves were constructed to assess the overall accuracy of suPAR and non-invasive fibrosis scoring systems to discriminate between fibrosis stage $0-2$ and fibrosis stage $3-4$. The ROC curve is a plot of sensitivity vs. specificity (1 - specificity) for all possible cut-off values. The most commonly used index of accuracy is the area under the ROC curve (AUROC), with values close to 1.0 indicating a high diagnostic accuracy.

A 2-tailed $p$ value of less than 0.05 was considered significant. All statistical analyses were performed with the SPSS Statistics 19-20 (IBM, Armonk, NY, USA) software. GraphPad Prism version 5.03 (GraphPad Software, San Diego, CA, USA) was used for figures.

\section{Ethics}

The study protocol was approved by the Linköping University Ethical Review Board (2010/406-31 and 02-454). 


\section{Results}

\section{Patient data}

In total, 82 individuals with NAFLD and 38 with HCV were enrolled in the study. 20 $(17 \%)$ patients had advanced fibrosis (stage 3-4); 16 of these were diagnosed with NAFLD and 4 with HCV. The remaining 62 patients had no or mild fibrosis (stage $0-2$ ). The age of the HC was similar to that of the HCV patients, whereas NAFLD patients were significantly older. Female gender was similar among HC and HCV patients, whereas NAFLD patients were males to a higher extent than HC. Characteristics of all patients and controls are given in Table I.

Increased suPAR levels in patients with chronic liver disease As demonstrated in Figure 1a, suPAR levels were significantly higher in NAFLD patients compared with HC $(4.8 \pm 1.8$ vs. $3.9 \pm 0.9 ; p<0.001)$; a similar difference was found between HCV patients and HC $(5.9 \pm 2.1$ vs. $3.9 \pm 0.9 ; p<0.001)$. In addition, HCV patients showed higher suPAR levels compared with NAFLD (5.9 \pm 2.1 vs. $4.8 \pm 1.8 ; p<0.002)$. As indicated in Figure 1b, regardless of background disease advanced fibrosis (stage 3-4) was significantly associated with higher suPAR levels in comparison with patients with no or mild fibrosis (stage $0-2)(6.2 \pm 1.9$ vs. $5.0 \pm 1.9 ; p=0.013)$. When restricting the study population to NAFLD patients exclusively, as depicted in Figure 1c, individuals with advanced fibrosis still showed higher suPAR levels compared with those with no or mild fibrosis $(5.7 \pm 1.8 v s .4 .6 \pm 1.7 ; p=0.025)$. A corresponding trend towards higher suPAR was found for the 4 patients with advanced fibrosis relative to the others with HCV $(7.8 \pm 1.3 v s .5 .6 \pm 2.1 ; p=0.050)$ as demonstrated in Figure $1 \mathrm{~d}$. Neither inflammation grade according to Batts and Ludwig, nor portal inflammation as defined by Knodell were 
associated with suPAR levels in the HCV group. Similarly, NAS was not associated with suPAR levels in the NAFLD group.

\section{Association of suPAR with routine laboratory measures and histopathology}

In NAFLD patients, suPAR levels were significantly associated with age, presence of diabetes mellitus, severity of fibrosis, PK-INR and QUICKI (Table III), but not with presence of metabolic syndrome, gender, BMI, hsCRP, ALP, AST, ALT, steatosis grade, NAS, or portal inflammation. In HCV patients, suPAR levels were associated with age, hsCRP, ALT, AST, ALP, but not with presence of diabetes mellitus, gender, BMI, PK-INR, fibrosis, steatosis grade or inflammation grade (Table III). suPAR levels did not significantly differ between HCV genotypes; and suPAR was not correlated with the amount of HCV-RNA.

suPAR versus severity of fibrosis

For all 120 patients, suPAR displayed an AUROC of 0.716 (Figure 2a) which was almost similar to the non-invasive fibrosis scoring systems, i.e. APRI (AUC 0.763) and GUCI (AUC 0.771). In NAFLD, an AUROC of 0.722 was achieved for suPAR. The corresponding figures for NAFLD fibrosis score, APRI, BARD, and GUCI were 0.773, 0.842, 0.709, and 0.856, respectively (Figure $2 \mathrm{~b}$ ). In HCV patients, an AUROC of 0.848 was achieved for suPAR compared with APRI 0.720, and GUCI 0.720.

\section{Discussion}

In the past decade, many investigators have proposed non-invasive replacements to liver biopsy, including either a single biochemical marker or a combination of tests, both 
serum markers and physical measurement of liver stiffness. ${ }^{1,4,7,8}$ Unfortunately, at the time of our study, transient elastography was unavailable at our center. While some investigators have focused on a combination of laboratory tests such as reversal of AST/ALT ratio or APRI, others have been searching for novel markers of fibrosis and inflammation. ${ }^{1}$ Despite sharing the common pathway for fibrosis, an imbalance between synthesis and degradation of extracellular matrix by activated HSC, there are currently no liver specific markers available that could accurately reflect liver fibrosis of different etiologies. ${ }^{1,37}$

Herein, suPAR was analyzed in two patient groups with chronic liver disease. We demonstrate that suPAR levels are associated with severity of fibrosis in both NAFLD and HCV. With only one exception (being hsCRP levels in HCV patients), inflammation including biomarkers and histopathology - was not associated with suPAR levels. This is consistent with our previous finding for SLE patients where serum suPAR correlated strongly with organ damage rather than inflammatory activity. ${ }^{26}$

The present study is the first to evaluate suPAR as a biomarker in NAFLD. The development of NAFLD is associated with insulin resistance, which is attributed to inflamed adipose tissue. ${ }^{10}$ In our well-characterized NAFLD cohort, we show that suPAR levels are higher in comparison with HC, whereas lower concentrations were found compared to HCV patients. In addition, we observed an association between suPAR levels and QUICKI as well as the presence of diabetes mellitus, whereas the association with metabolic syndrome did not reach statistical significance $(p=0.14)$. Thus, a connection between suPAR and the degree of inflammation cannot be excluded in NAFLD. In line with this observation, it was recently shown that suPAR levels are 
associated with incident diabetes among overweight but not obese individuals with impaired glucose regulation. ${ }^{38}$ Lifestyle factors, such as smoking and physical activities, as well as age have an effect on baseline suPAR levels also in a healthy population. ${ }^{39}$ As expected, age was associated with suPAR in both patient groups (Table III); this is important to take into account in the assessment of measurements in any patient group.

Although we here analyzed a limited number of HCV patients, suPAR was higher in patients with advanced compared to mild fibrosis (Figure 1b). Indeed, suPAR has previously been evaluated as a non-invasive fibrosis marker in HCV. Berres and colleagues concluded that serum suPAR has a high diagnostic specificity and sensitivity to differentiate mild (stage 1-2) from severe (stage 3-4) fibrosis with an AUROC of $0.774 . .^{25}$ Zimmermann et al presented similar results and pointed out that suPAR levels above $9 \mathrm{ng} / \mathrm{mL}$ could constitute a strong predictor of mortality or the need for liver transplantation. ${ }^{23}$ Thus, our results regarding HCV are clearly in line with previous observations. Considering the question raised by Dr Filik, we did not find any correlation between viral load and suPAR levels. ${ }^{40}$ Thus, it is possible that suPAR could be attributed to liver fibrosis per se rather than due to the viral infection.

We found a significant moderate correlation between suPAR concentration and fibrosis stage, as well as a difference between mild and advanced fibrosis (Figure 1b). The NAFLD fibrosis score, APRI, BARD and GUCI are all commonly used scores for estimating the severity of fibrosis. ${ }^{41}$ APRI and GUCI were primarily developed for HCV. Herein, these scores were compared with suPAR alone regarding performance in predicting severity of fibrosis. Intriguingly, for all 120 patients, suPAR was essentially as useful as APRI and GUCI. In the 82 NAFLD patients, NAFLD fibrosis score, APRI and GUCI 
performed better than suPAR, but suPAR was at least as good as BARD. The question whether suPAR gives additional information regarding severity of fibrosis was beyond the scope of this paper. One possibility to further investigate this would however be to create a fibrosis score where suPAR is included as a separate component and apply this to larger study populations. One advantage by analyzing suPAR is the stability of the molecule as it is not substantially affected by freeze/thaw cycles. In addition, the interday variations are negligible and the concentration is not influenced by dietary intake. ${ }^{42}$

Whether suPAR constitutes an epiphenomenon or if it plays an active role in the development of liver fibrosis remains open. In the liver, activation of the innate immune system has a critical role in maintaining homeostasis and liver regeneration, as well as disease pathogenesis, acting in a cooperative rather than independent fashion. ${ }^{43}$ To our knowledge, there are no published data on how suPAR affects the activation of HSC and potential effects on the balance between synthesis and degradation of extracellular matrix. However, Koch et al recently demonstrated that the human HSC line LX2 has the capability to spontaneously secrete suPAR during cultivation although the levels were low. ${ }^{44}$ In addition, HSC from healthy donors have been shown to produce uPAR, and the addition of UPA enhanced the chemotactic and chemoinvasive properties of the HSC. 45 The role of innate immune components such as mast cells, neutrophils, macrophages, NK cells, complement pathway proteins, secreted pattern-recognition receptors, and acute phase reactants have been implicated in liver disease. ${ }^{46,47}$ To induce inflammation and subsequent organ damage, these cells need to cross the blood barrier and be directed to the tissue. The classical chemoattractant for neutrophils is interleukin-8. ${ }^{48}$ However, it has been shown that suPAR fragment $\mathrm{D}_{\text {II-III }}$ can act as a chemoattractant for neutrophils and monocytes. ${ }^{49-51}$ Neutrophils and monocytes are also important for the 
release of suPAR, which could induce further recruitment of inflammatory cells and perpetuate the inflammation into a vicious circle. ${ }^{44,52}$

To conclude, this is the first study to describe an association between circulating suPAR and severity of fibrosis in NAFLD. In addition, we confirmed previous reports on HCV demonstrating a corresponding association. Accordingly, suPAR appears to reflect severity of fibrosis rather than inflammation in these conditions, and thus constitute a promising biomarker candidate. ${ }^{53}$ Further prospective studies will have to address the question if suPAR provides additional information compared to the present noninvasive scoring systems for liver fibrosis.

\section{Acknowledgments}

Conflict of interests: None.

Disclosure statement: Nothing to declare.

The authors thank research nurse Liselott Lindvall and the clinicians at Linköping University Hospital. Dr Jonas Wetterö is acknowledged for comments on the manuscript. This work was supported by grants from the Swedish Society for Medical Research, the Professor Nanna Svartz foundation, the King Gustaf V 80-year foundation, the Swedish Society of Medicine, the Swedish Rheumatism Association, the County Council of Östergötland, and the Medical Research Council of Southeast Sweden. All authors have (i) approved the final version of the manuscript, (ii) read the journal's policy on conflicts of interest, and (iii) read the journal's authorship agreement. 


\section{References}

1. Baranova A, Lal P, Birerdinc A, Younossi ZM. Non-invasive markers for hepatic fibrosis. BMC Gastroenterol 2011;11:91

2. Feldman: Sleisenger and Fordtran's Gastrointestinal and Liver Disease, 9th ed. 2010 Saunders: an imprint of Elsevier

3. Cadranel J, Rufat P, Degos F. Practices of liver biopsy in France: results of a prospective nationwide survey. For the Group of Epidemiology of the French Association for the Study of the Liver (AFEF). Hepatology 2000;32:477-81

4. Abdi W, Millan JC, Mezey E. Sampling variability on percutaneous liver biopsy. Arch Intern Med 1979;139:667-69

5. Regev A, Berho M, Jeffers LJ, et al. Sampling error and intraobserver variation in liver biopsy in patients with chronic HCV infection. Am J Gastroenterol 2002;97:2614-18

6. Castera L. Noninvasive methods to assess liver disease in patients with hepatitis B or C. Gastroenterology 2012;142:1293-1302

7. Holmberg SD, Lu M, Rupp LB, et al. Noninvasive serum fibrosis markers for screening and staging chronic hepatitis $C$ virus patients in a large US cohort. Clin Infect Dis 2013;57:240-46

8. Ray K. NAFLD - the next global epidemic. Nat Rev Gastroenterol Hepatol $2013 ; 10: 621$

9. Yilmaz Y. Biomarkers for early detection of non-alcoholic steatohepatitis: implications for drug development and clinical trials. Curr Drug Targets 2013;14:1357-366

10. Castera L, Vilgrain V, Angulo P. Noninvasive evaluation of NAFLD. Nat Rev Gastroenterol Hepatol 2013;10:666-75

11. Wilhelm OG, Wilhelm S, Escott GM, et al. Cellular glycosylphosphatidylinositolspecific phospholipase D regulates urokinase receptor shedding and cell surface expression. J Cell Physiol 1999;180:225-35

12. Thunø M, Macho B, Eugen-Olsen J. suPAR: the molecular crystal ball. Dis Markers 27:157-72 
13. Barnathan ES, Kuo A, Kariko K, et al. Characterization of human endothelial cell urokinase-type plasminogen activator receptor protein and messenger RNA. Blood 1990;76:1795-806

14. Wohn KD, Kanse SM, Deutsch V, Schmidt T, Eldor A, Preissner KT. The urokinasereceptor (CD87) is expressed in cells of the megakaryoblastic lineage. Thromb Haemost 1997;77:540-47

15. Plesner T, Ralfkiaer E, Wittrup M, et al. Expression of the receptor for urokinasetype plasminogen activator in normal and neoplastic blood cells and hematopoietic tissue. Am J Clin Pathol 1994;102:835-41

16. Vassalli JD, Baccino D, Belin D. A cellular binding site for the Mr 55,000 form of the human plasminogen activator, urokinase. J Cell Biol 1985;100:86-92

17. Nykjaer A, Møller B, Todd RF III, et al. Urokinase receptor: an activation antigen in human T lymphocytes. J Immunol 1994;152:505-16

18. Kirchheimer JC, Nong YH, Remold HG. IFN-gamma, tumor necrosis factor-alpha, and urokinase regulate the expression of urokinase receptors on human monocytes. J Immunol 1988;141:4229-34

19. Chavakis T, Willuweit AK, Lupu F, Preissner KT, Kanse SM. Release of soluble urokinase receptor from vascular cells. Thromb Haemost 2001;86:686-93

20. Sidenius N, Sier CF, Blasi F. Shedding and cleavage of the urokinase receptor (UPAR): identification and characterisation of UPAR fragments in vitro and in vivo. FEBS Lett 2000;475:52-56

21. Backes Y, van der Sluijs KF, Mackie DP, et al. Usefulness of suPAR as a biological marker in patients with systemic inflammation or infection: a systematic review. Intensive Care Med 2012;38:1418-28

22. Haupt TH, Petersen J, Ellekilde G, et al. Plasma suPAR levels are associated with mortality, admission time, and Charlson Comorbidity Index in the acutely admitted medical patient: a prospective observational study. Crit Care 2012;16:R130

23. Zimmermann HW, Koch A, Seidler S, Trautwein C, Tacke F. Circulating soluble urokinase plasminogen activator is elevated in patients with chronic liver disease, discriminates stage and aetiology of cirrhosis and predicts prognosis. Liver Int 2012;32:500-509 
24. Andersen ES, Ruhwald M, Moessner B, et al. Twelve potential fibrosis markers to differentiate mild liver fibrosis from cirrhosis in patients infected with chronic hepatitis C genotype 1. Eur J Clin Microbiol Infect Dis 2011;30:761-66

25. Berres ML, Schlosser B, Berg T, Trautwein C, Wasmuth HE. Soluble urokinase plasminogen activator receptor is associated with progressive liver fibrosis in hepatitis C infection. J Clin Gastroenterol 2012;46:334-38

26. Enocsson H, Wetterö J, Skogh T, Sjöwall C. Soluble urokinase plasminogen activator receptor levels reflect organ damage in systemic lupus erythematosus. Transl Res 2013;162:287-96

27. Batts KP, Ludwig J. Chronic hepatitis. An update on terminology and reporting. Am J Surg Pathol 1995;19:1409-17

28. Knodell RG, Ishak KG, Black WC, et al. Formulation and application of a numerical scoring system for assessing histological activity in asymptomatic chronic active hepatitis. Hepatology 1981;1:431-35

29. Kleiner D, Brunt E, Van Natta M, et al. Design and validation of a histological scoring system for nonalcoholic fatty liver disease. Hepatology 2005;41:1313-21

30. Franzén LE, Ekstedt M, Kechagias S, Bodin L. Semiquantitative evaluation overestimates the degree of steatosis in liver biopsies: a comparison to stereological point counting. Mod Pathol 2005;18:912-16

31. Angulo P, Hui JM, Marchesini G, et al. The NAFLD fibrosis score: a noninvasive system that identifies liver fibrosis in patients with NAFLD. Hepatology $2007 ; 45: 846-54$

32. Wai CT, Greenson JK, Fontana RJ, et al. A simple noninvasive index can predict both significant fibrosis and cirrhosis in patients with chronic hepatitis C. Hepatology 2003;38:518-26

33. Harrison SA, Oliver D, Arnold HL, et al. Development and validation of a simple NAFLD clinical scoring system for identifying patients without advanced disease. Gut 2008;57:1441-47

34. Islam S, Antonsson L, Westin J, et al. Cirrhosis in hepatitis C virus-infected patients can be excluded using an index of standard biochemical serum markers. Scand J Gastroenterol 2005;40:867-72 
35. Katz A, Nambi SS, Mather K, et al. Quantitative insulin sensitivity check index: a simple, accurate method for assessing insulin sensitivity in humans. J Clin Endocrinol Metab 2000;85:2402-10

36. Expert panel on detection, evaluation, and treatment of high blood cholesterol in adults (Adult treatment panel III). Executive summary of the third report of the national cholesterol education program (NCEP). JAMA 2001;285:2486-97

37. Schuppan D, Afdhal NH. Liver cirrhosis. Lancet 2008;371:838-51

38. Heraclides A, Jensen TM, Rasmussen SS, et al. The pro-inflammatory biomarker soluble urokinase plasminogen activator receptor (suPAR) is associated with incident type 2 diabetes among overweight but not obese individuals with impaired glucose regulation: effect modification by smoking and body weight status. Diabetologia 2013;56:1542

39. Eugen-Olsen J, Andersen O, Linneberg A, et al. Circulating soluble urokinase plasminogen activator receptor predicts cancer, cardiovascular disease, diabetes and mortality in the general population. J Intern Med 2010;268:296-308

40. Filik L. Soluble urokinase plasminogen activator receptor in chronic hepatitis due to hepatitis C virus. J Clin Gastroenterol 2012;46:346-7

41. Usluer G, Erben N, Aykin N, et al. Comparison of non-invasive fibrosis markers and classical liver biopsy in chronic hepatitis C. Eur J Clin Microbiol Infect Dis 2012;31:1873-78

42. Botha S, Fourie CM, Schutte R, Kruger A, Schutte AE. Associations of suPAR with lifestyle and cardiometabolic risk factors. Eur J Clin Invest 2014;44:619-26

43. Gao B, Jeong WI, Tian Z. Liver: An organ with predominant innate immunity. Hepatology 2008;47:729-36

44. Koch A, Zimmermann HW, Gassler N, et al. Clinical relevance and cellular source of elevated soluble urokinase plasminogen activator receptor (suPAR) in acute liver failure. Liver Int 2014, Feb 28. doi: 10.1111/liv.12512 [Epub ahead of print]

45. Fibbi G, Pucci M, Grappone C, et al. Functions of the fibrinolytic system in human Ito cells and its control by basic fibroblast and platelet-derived growth factor. Hepatology 1999;29:868-78

46. Rensen SS, Slaats Y, Nijhuis J, et al. Increased hepatic myeloperoxidase activity in obese subjects with nonalcoholic steatohepatitis. Am J Pathol 2009;175:1473-82 
47. Rensen SS, Slaats Y, Driessen A, et al. Activation of the complement system in human nonalcoholic fatty liver disease. Hepatology 2009;50:1809-17

48. Lalor PF, Faint J, Aarbodem Y, Hubscher SG, Adams DH. The role of cytokines and chemokines in the development of steatohepatitis. Semin Liver Dis 2007;27:17393

49. Fazioli F, Resnati M, Sidenius N, Higashimoto Y, Appella E, Blasi F. A urokinasesensitive region of the human urokinase receptor is responsible for its chemotactic activity. EMBO J 1997;16:7279-86

50. Pliyev BK. Activated human neutrophils rapidly release the chemotactically active $D_{2} D_{3}$ form of the urokinase-type plasminogen activator receptor (uPAR/CD87). Mol Cell Biochem 2009;321:111-22

51. Mondino A, Blasi F. UPA and uPAR in fibrinolysis, immunity and pathology. Trends Immunol 2004;25:450-55

52. Pliyev BK, Menshikov MY. Release of the soluble urokinase-type plasminogen activator receptor (suPAR) by activated neutrophils in rheumatoid arthritis. Inflammation 2010;33:1-9

53. Vasarhelyi B. Soluble urokinase plasminogen activator receptor, the candidate prophetic biomarker in severe inflammatory response syndrome. J Intern Med 2014, Apr 15. doi: 10.1111/joim.12259 [Epub ahead of print] 


\section{Legends}

Figure 1: Serum suPAR concentrations in NAFLD and HCV patients, and healthy controls (HC) analyzed by ELISA (a). ${ }^{* *} p<0.001 ;{ }^{* *} p<0.002$. Serum suPAR concentrations according to fibrosis stage; mild (stage 0-2) and advanced (stage 3-4), demonstrated for all patients (b), NAFLD patients only (c) and HCV patients only (d). Open circles represent NAFLD and filled circles HCV. Mean concentrations \pm SD are indicated by horizontal bars.

Figure 2: Serum soluble urokinase plasminogen activator receptor (suPAR) concentration as a predictor of severity of fibrosis in comparison with other commonly used non-invasive fibrosis scoring systems in all 120 patients (a), and in the 82 NAFLD patients exclusively (b). Area under the ROC curve (AUROC) values are given in the main text. 


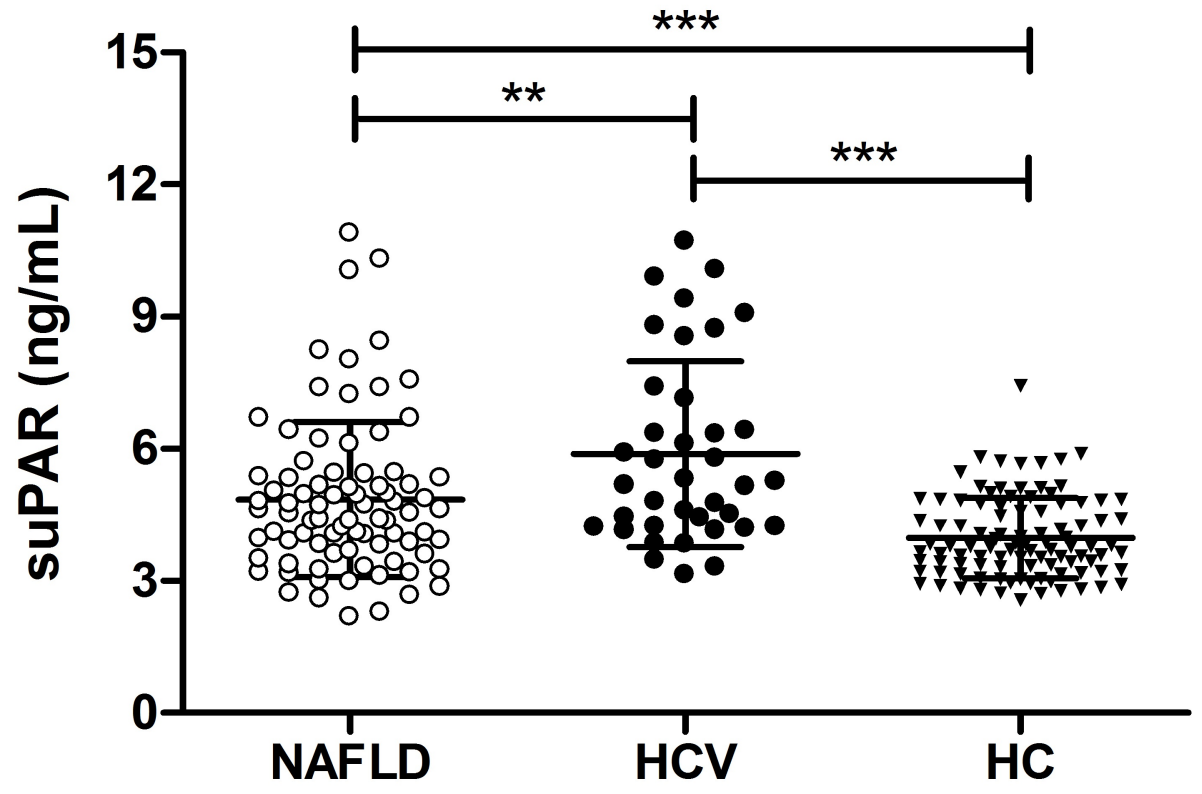




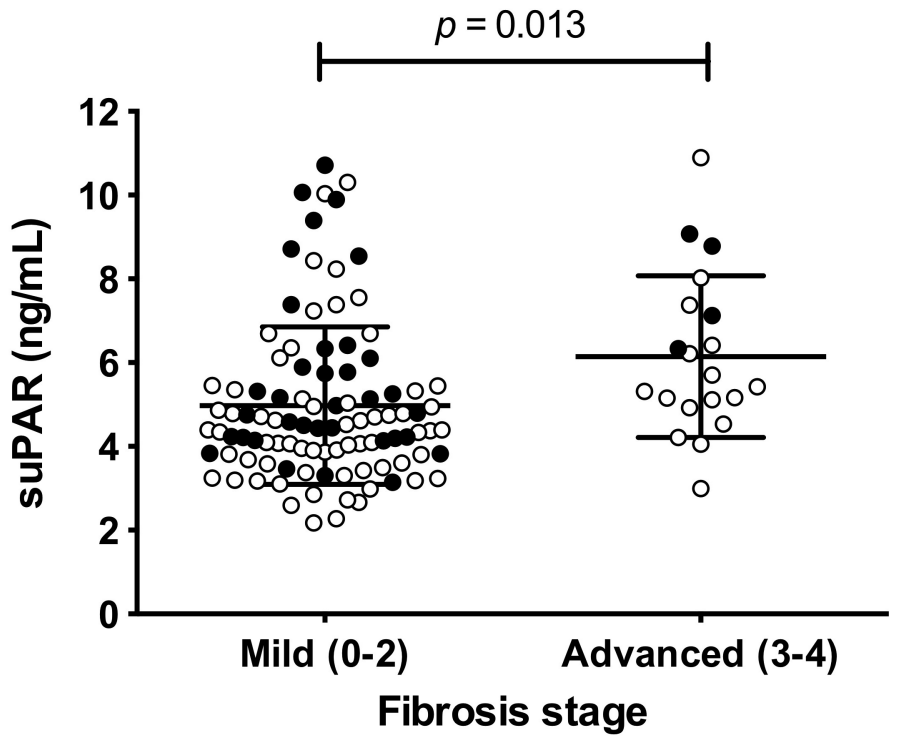




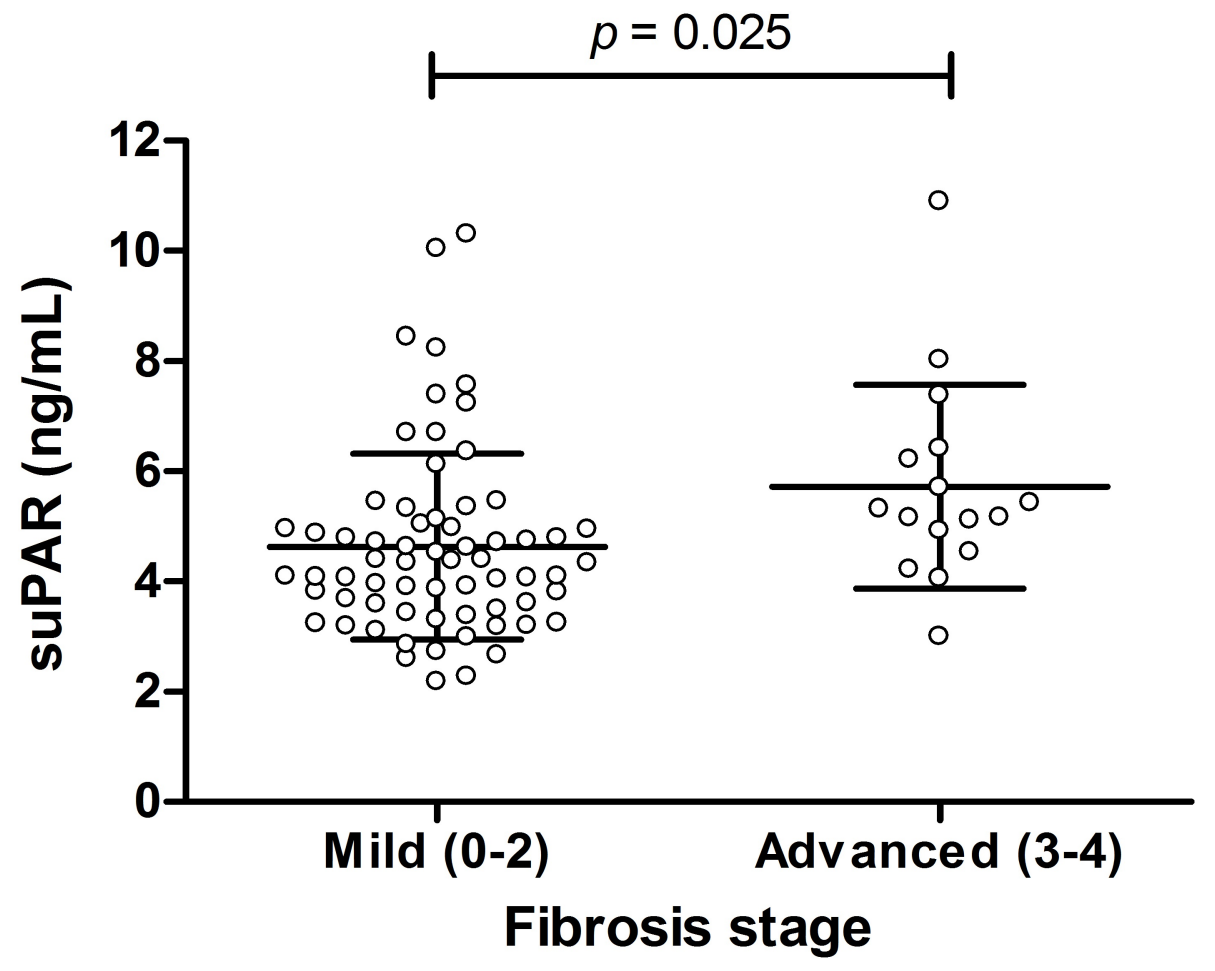




$$
p=0.050
$$

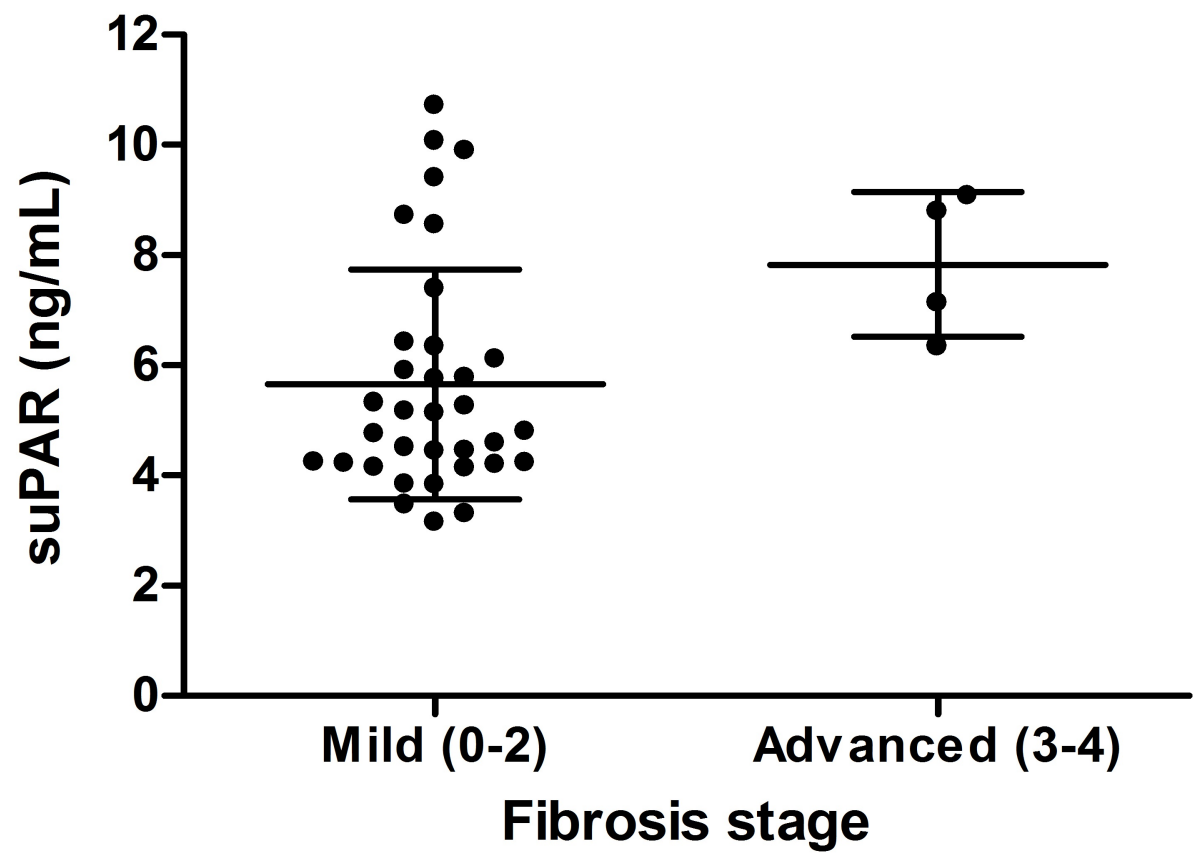


Table I. Characteristics of the non-alcoholic fatty liver disease (NAFLD) and hepatitis C virus (HCV) infected study populations, as well as the healthy controls (HC).

\begin{tabular}{|c|c|c|c|c|}
\hline & NAFLD & HCV & $\mathrm{HC}$ & \\
\hline Patient data & $(n=82)$ & $(n=38)$ & $(n=100)$ & $p=$ \\
\hline Age (years) & $59.8 \pm 11.7$ & $45.3 \pm 15.8$ & $45.8 \pm 11.9$ & $<0.001$ \\
\hline Gender (male) & $55(67 \%)$ & $22(58 \%)$ & 50 (50\%) & 0.02 \\
\hline BMI & $28.9 \pm 4.4$ & $24.9 \pm 4.1$ & $*$ & 0.025 \\
\hline Diabetes mellitus & $42(51 \%)$ & $1(2.6 \%)$ & $0(0 \%)$ & $<0.001$ \\
\hline Platelet count $\left(10^{9} / \mathrm{L}\right)$ & $231 \pm 66$ & $247 \pm 74$ & $*$ & 0.24 \\
\hline Albumin (g/L) & $42 \pm 3.8$ & $41 \pm 3.8$ & $*$ & 0.13 \\
\hline hsCRP (mg/L) & $3.3 \pm 5.0$ & $1.3 \pm 1.9$ & $*$ & 0.021 \\
\hline Bilirubin $(\mu \mathrm{mol} / \mathrm{L})$ & $13 \pm 5.4$ & $15 \pm 23$ & $*$ & 0.62 \\
\hline ALP $(\mu \mathrm{kat} / \mathrm{L})$ & $3.2 \pm 2.2$ & $2.4 \pm 1.4$ & $*$ & 0.057 \\
\hline AST $(\mu \mathrm{kat} / \mathrm{L})$ & $0.65 \pm 0.28$ & $1.6 \pm 2.6$ & $*$ & 0.001 \\
\hline ALT ( $\mu$ kat/L) & $1.1 \pm 0.67$ & $2.6 \pm 3.2$ & $*$ & $<0.001$ \\
\hline GGT $(\mu \mathrm{kat} / \mathrm{L})$ & $1.3 \pm 1.2$ & $1.2 \pm 1.3$ & $*$ & 0.72 \\
\hline PK-INR & $1.0 \pm 0.09$ & $1.0 \pm 0.09$ & $*$ & 0.31 \\
\hline
\end{tabular}


Table II. The following formulas were used for calculation of the fibrosis scoring systems.

\begin{tabular}{|c|c|}
\hline $\begin{array}{l}\text { NAFLD fibrosis } \\
\text { score }^{31}\end{array}$ & $\begin{array}{l}-1.675+0.037 \times \text { age }(\text { years })+0.094 \times \mathrm{BMI}\left(\mathrm{kg} / \mathrm{m}^{2}\right)+1.13 \times \text { Impaired fasting glucose/Diabetes } \\
(\text { yes }=1, \mathrm{no}=0)+0.99 \times \mathrm{AST} / \mathrm{ALT} \text { ratio }-0.013 \times \text { Platelet count }\left(10^{9} / \mathrm{L}\right)-0.66 \times \text { albumin }(\mathrm{g} / \mathrm{dL})\end{array}$ \\
\hline $\mathrm{APRI}^{32}$ & AST level /ASTuLn $\times 100 /$ Platelet count $\left(10^{9} / \mathrm{L}\right)$ \\
\hline BARD-score ${ }^{33}$ & $\mathrm{BMI} \geq 28=1 \mathrm{p}, \mathrm{AST} / \mathrm{ALT}$ ratio $\geq 0.8=2 \mathrm{p}$, Diabetes $=1 \mathrm{p}$ \\
\hline $\mathbf{G U C l}^{34}$ & AST $(\mu \mathrm{kat} / \mathrm{L}) / \mathrm{AST}_{\text {ULN }}(\mu \mathrm{kat} / \mathrm{L}) \times$ prothrombin-INR $\times 100 /$ Platelet count $\left(10^{9} / \mathrm{L}\right)$ \\
\hline
\end{tabular}


Table III. Correlations between serum suPAR levels and disease variables using Pearson.

\begin{tabular}{|c|c|c|c|c|}
\hline Disease & & & & \\
\hline Factor & $r=$ & $p=$ & $r=$ & $p=$ \\
\hline Age (years) & 0.43 & $<0.0001$ & 0.39 & 0.017 \\
\hline BMI & & NS & & NS \\
\hline hsCRP & & NS & 0.36 & 0.027 \\
\hline ALP & & NS & 0.35 & 0.041 \\
\hline AST & & NS & 0.37 & 0.023 \\
\hline ALT & & NS & 0.36 & 0.026 \\
\hline PK-INR & 0.24 & 0.030 & & NS \\
\hline HCV-RNA & & $*$ & & NS \\
\hline QUICKI & -0.32 & 0.016 & & * \\
\hline Fibrosis stage & 0.29 & 0.008 & & NS \\
\hline Inflammation grade & & NS & & NS \\
\hline NAS & & NS & & $*$ \\
\hline Steatosis (quantitative) & & NS & & $*$ \\
\hline
\end{tabular}

\footnotetext{
* Not analyzed

NS = not significant
} 